\title{
La relazione fraterna come dimensione paritaria dei rapporti umani
}

\section{Fraternal relationship as equal dimension of hu- man rapports}

\author{
Fabio Frisone*
}

Salvatore Settineri**

Emanuele Maria Merlo***

Recebido: 09/04/2018. Aprovado: 19/04/2018.

Sommario: Il tema della fraternità risulta essere un fatto fondativo nella relazionalità matura, nella misura in cui coinciderebbe con la avvenuta maturazione psichica. Nell'articolazione delle figure affettive di riferimento e nella loro successione integrativa, infatti, si esplica l'ammissione dell'Altro nel passaggio dalla primità alla secondità e alla terzeità e, dunque, nel superamento delle criticità di ogni fase. Ciò comporta che l'ammissione delle necessità dell'Altro, la comprensione e la riconciliazione, possano essere considerate come l'esito di maturazioni di immagini e rappresentazioni intrapsichiche legate ad un tema affettivo. La fenomenologia delle immagini e dell'affettività, le loro successioni e, con riferimento agli esiti patologici, la loro immaturità consentono la comprensione. Con un ponte tra la letteratura teologica e la clinica psicologica risulta comprensibile l'esplicarsi di processi che si ripercuotono su di noi a partire dalla vita quotidiana per finire alla vita politica. In tal senso, un tema così fine come l'ottica della

* Laurea Triennale in Scienze e Tecniche Psicologiche (L-24), Università degli Studi di Messina, 2012. Laurea Magistrale in Fondamenti e Prospettive di una Cultura dell'Unità, specializzazione in Studi Politici, Istituto Universitario Sophia, 2015. Laurea Magistrale in Psicologia (LM-51), Università degli Studi di Messina, 2017.

E-mail: fabio.frisone@hotmail.com

** Dottore in Medicina e Chirurgia, Università degli Studi di Messina, 1977. Professore Associato in Psicologia Clinica, Dipartimento di Scienze Biomediche, Odontoiatriche e delle Immagini Morfologiche e Funzionali, Università degli Studi di Messina.

E-mail: settineri@unime.it

*** Laurea Triennale in Scienze e Tecniche Psicologiche (L-24), Università degli Studi di Messina, 2015. Laurea Magistrale in Psicologia (LM-51), Università degli Studi di Messina, 2017. Dottorando di ricerca, Dipartimento di Scienze Cognitive, Psicologiche, Pedagogiche e degli Studi Culturali, Università degli Studi di Messina.

E-mail: emerlo@unime.it 
considerazione dell'Altro attraverso la lente della fraternità, consente di chiarire come alla maturità psichica corrisponda una matura considerazione dell'alterità, comprensiva al contempo della uguaglianza nel diritto e delle evidenti differenze.

Parole chiave: Fraternità. Psicologia della fraternità. Rapporti umani

Resumo: O tema da fraternidade acaba sendo um fato fundador da relacionalidade humana, na medida em que coincide com a completa maturidade psíquica. $\mathrm{Na}$ articulação das figuras afetivas de referência e na sua sucessão integrativa, de fato, se explica a admissão do Outro na passagem da primidade à secundidade e, então, à terceidade $e$, portanto, na superação dos pontos críticos de cada fase. Isso implica que a integração de necessidades do Outro, a compreensão e a reconciliação, podem ser consideradas como o resultado do amadurecimento de imagens e representações intrapsíquicas ligadas a um tema afetivo. A fenomenologia das imagens e da afetividade, as suas sucessões e, com referência aos êxitos patológicos, a sua imaturidade consentem a compreensão. Com uma ponte entre a literatura teológica e a clínica psicológica torna-se compreensível a explicação de processos que repercutem sobre nós desde a vida cotidiana até a vida política. Neste sentido, um tema como a ótica da consideração do Outro através da lente da fraternidade, permite esclarecer como à maturidade psíquica corresponda uma madura consideração da alteridade, que compreende ao mesmo tempo a igualdade de direitos e as evidentes diferenças.

Palavras-chave: Fraternidade. Psicologia da fraternidade. Relacionamentos humanos.

\section{Introduzione}

Quando ci si interroga su temi attraverso cui portare un contributo per l'approfondimento di dinamiche psichiche rilevanti, è a partire dalla trasversalità e dall'impatto che tali fenomeni riescono ad alimentare che scaturisce la possibilità di vivere esperienze di rilevante matrice interiore. Partendo da tale presupposto, appare difficile non considerare l'idea di una esperienza umana che risulta intrinsecamente connessa allo stare bene con gli altri. Volendo, dunque, approfondire i temi sulla dimensione relazionale, occorre approfondire un particolare tipo di paradigma, quello della fraternità, a partire dal contributo di un libro di Antonio Maria Baggio (2012), intitolato Caino e i suoi fratelli.

È bene chiarire sin da subito che la relazione fraterna di cui si parlerà oggi viene intesa come un «"luogo" conoscitivo [...] nel quale approfondire l'interpretazione delle relazioni umane e, in particolare, l'interpretazione dei conflitti» ${ }^{1}$ non soltanto nella sfera privata, quanto piuttosto nello spazio pubblico.

1 BAGGIO, A. M. La fraternità antagonista. L'interpretazione freudiana e la fondazione della società egualitaria e conflittuale. In: Caino e i suoi fratelli. II fondamento relazionale nella politica e nel diritto, a cura di A. M. Baggio, Città Nuova, Roma 2012, p. 19. 
La relazione fraterna può esemplificare una radicale conflittualità ma, allo stesso tempo, essa attesta un nucleo di co-appartenenza allo stesso contesto. Da ciò scaturisce la possibilità di uso della forza derivante dal legame, che prevede la considerazione dell'altro da declinare alla domanda relativa alla propria identità. Da questo punto di vista, la rilevanza della rappresentazione di cosa - e dunque immagine - e di parola - e dunque idea -, contiene il fatto relazionale che si muove polarmente dall'uguaglianza nel diritto alla differenza inevitabile.

«Due che non si sono scelti, così come la condizione umana - di essere uomini non da soli, ma tra gli altri uomini - non si può scegliere: la fraternità si presenta come l'espressione archetipica originaria della realtà umana come realtà relazionale tra pari»». ${ }^{2}$

Potrebbe, inoltre, risultare importante riflettere già adesso sul fatto che la relazione fraterna, inevitabilmente, implica «il correlativo concetto di paternità e, con esso, il principio di autorità che gli è collegato» ${ }^{3}$.

\section{Psicodinamica delle origini della fraternità}

Già dagli esordi della considerazione di Freud in Totem e tabù ${ }^{4}$, si apre la riflessione psicoanalitica sulla fraternità come prototipo di rappresentazioni afferenti allo sviluppo della vita sociale rispetto alle figure genitoriali primarie, base delle triangolazioni future ${ }^{5}$; ovvero, come possibilità di una esperienza di un "non-Io", che conferisca l'opportunità di sviluppo dell'alterità e di mitigazione di temi narcisistici tramite investimento oggettuale ${ }^{6}$.

La conoscenza della mitologia quale considerazione analitica dell'origine propone, in questi termini, la considerazione di fatti simbolici di periodi umani in cui coscienza e inconscio risultano unificati e il naturalismo fenomenico non rimanda all' evento stesso, quanto all' espressione

2 IVi, p. 23.

$3 \quad$ Ivi, p. 20.

4 FREUD, S. Totem e Taboo (1913). Opere di Sigmund Freud (1912-1914). Opere, VII, Torino, Bollati Boringhieri, 2000.

5 BRUSSET, A. La lien fraternel et la psychanalyse, Psychanalyse à l'Universitè, 12, n. 45, Paris, AUREPP, 1987.

6 LECHARTIER-ATLAN, C. (2008). Frères et sœurs: une introduction. Revue française de psychanalyse, 72(2), 331-338. 
inconscia stessa ${ }^{7}$. Possiamo anzitutto notare come nei miti che raccontano le origini di molte civiltà, le coppie fraterne abbiano spesso avuto un ruolo cruciale nel definire l'architettura culturale, lo sviluppo umano e la conseguente organizzazione psichica.

Occorre, inoltre, precisare la distinzione che caratterizza due diverse tipologie di fratelli: i fratelli possono essere gemelli, sia l'uno maggiore e l'altro minore. All'interno della dinamica gemellare si nota la duplicità dell'universo: i gemelli possono sia collaborare che combattere, ad ogni modo rappresentano la sorgente del nuovo che potrà nascere, in maniera armonica o traumatica ${ }^{8}$. Esempi in letteratura classica, come per quanto riguarda le figure dei Diòscuri Càstore e Polluce, ne evidenziano la funzione protettrice, tale del conferimento, da parte di Poseidone, del dominio del vento e del mare, e dell'immortalità, da parte di Zeus, successivamente alla lotta con Teseo per il rapimento della sorella Elena.

Un altro esempio riguarda il Messico: presso gli indios Pueblo, le divinità della sera e del mattino risultano entrambe protettrici. Presso gli Irochesi, invece, il gemello buono rappresenta il bene come principio di ordine all'interno dell'accampamento, mentre quello cattivo esercita il suo potere all'esterno'.

Un caso di fondazione di città legato ad una fraternità non gemellare risulta quello di Caino ed Abele laddove, come primo esempio, assistiamo alle dinamiche di un complesso fraterno che assume ora rilevanza autonoma. Rispetto al modello gemellare, il racconto del libro della Genesi sottolinea l'aspetto gerarchico, mette in evidenza il fatto che Dio possa decidere di cambiare i disegni degli uomini, senza badare troppo ai significati simbolici della primogenitura, profondamente radicati nella stessa cultura che ha composto il libro della Genesi. Il conflitto qui nasce proprio perché la gerarchia prestabilita viene ribaltata, dato che Dio preferisce il fratello minore ${ }^{10}$.

7 JUNG, C. G. \& KARÖLYI, K. (1948), Prolegomeni allo studio scientifico della mitologia, trad. it. Torino, Einaudi.

8 Cfr. BAGGIO, A. M. La fraternità antagonista. L'interpretazione freudiana e la fondazione della società egualitaria e conflittuale, In: Caino e i suoi fratelli. Il fondamento relazionale nella politica e nel diritto, a cura di A.M. Baggio, Città Nuova, Roma 2012, pp. 21-22.

$9 \quad$ Ivi, p. 22.

$10 \quad$ Ivi, p. 23. 
La fraternità come proposizione non della morale quanto della giustizia egalitaria e della sua rivendicazione ${ }^{11}$, può essere considerata a partire da un esempio il cui esito è nefasto, occasione di strutturazione e superamento di un tema della gelosia dell'oggetto, dell'invidia e, dunque, del desiderio non tanto dell'oggetto, quanto del desiderio stesso $^{12}$ dell'Altro. La temporalità e la possibilità di radicamento di tali fenomeni rappresentazionali maladattivi costituirebbe la formazione e la polarizzazione affettiva complessuale. Il cambiamento a fronte della relazione basata sulla reciprocità risulterebbe trasversale e legato contemporaneamente sia al principio di piacere, che al principio di realtà ${ }^{13}$.

In tal senso, la considerazione della rilevanza di un alter-ego potrebbe essere considerata alla stregua di uno specchio dell'identità, esercitando una funzione di modulazione dei fenomeni di proiezione della sfera intrafamiliare, promuovendo fenomeni imitativi, di accomodamento e di considerazione della passività. Da questo punto di vista, la funzione della rappresentazione fraterna favorirebbe le successive triangolazioni, che potrebbero risultare non sufficientemente protratte se limitate alla figura paterna. Allo stesso modo, produrrebbe una articolazione progressiva in riferimento alla posizione schizo-paranoide e depressiva, promuovendo la considerazione dei sentimenti di invidia primaria. Il superamento dunque del complesso fraterno, come suggerito da Brusset in merito al rapporto tra l'esperienza fraterna e la psicoanalisi, promuoverebbe la prevenzione di quella esperienza psicopatologica da fissazione alla relazione fraterna ${ }^{14}$, le cui espressioni in senso analitico sono dovute al nucleo affettivo-emotivo, attorno al quale le immagini della fraternità trarrebbero significato ${ }^{15}$.

Un fattore psichico, dunque, al di sopra della potenza energetica delle intenzioni coscienti, capace di generare uno stato di costrizione al pensiero reiterativo e all'azione.

11 MALLET, J. (2008). Une théorie de la paranoïa. Revue française de psychanalyse, 72(2), 341-346.

12 RECALCATI, M. (2012). Ritratti del desiderio, Raffaello Cortina Editore.

13 BRUSSET, B. (2008). Le lien fraternel et la psychanalyse, Revue française de psychanalyse, 72(2), 347-382.

14 LACAN J. (1932). De la psychose paranoiaque dans ses rapports avec la personalitè, Paris, Le Francoise; rééd. Paris, Le Seuil, 1975.

15 JUNG, C.G. Considerazioni generali sulla Teoria dei Complessi (1934). Opere di Jung, vol. 8.1976, Torino, Bollati, Borringhieri. 


\section{Fenomenologia identitaria e alterità}

Caino agricoltore ed Abele pastore si dedicano ad attività differenti, suggerendo il tema antropologico della stanzialità a confronto con il nomadismo. Nel piano originario del Creatore l'idea era quella di creare una convivenza armonica ${ }^{16}$. Ciononostante, si arriva ad un conflitto che porta all'esclusione dell'altro.

La conflittualità, dunque, si esprime attraverso l'impossibilità di almeno uno dei due di ammettere l'esistenza altrui, nel diniego rigido di ammetterne la presenza, nella rilevazione dell'Altro in quanto minaccia nell'affermazione delle dinamiche del Sé. Sorge quindi il tema dell'eliminazione, della distruttività del Thanatos che si esprime nell'acting che abbatte la polarità dell'uguale e contemporaneamente diverso, che denigra la responsabilità sull'Altro. La negazione di queste dinamiche rappresenta la negazione della fraternità. Come suggerito da Lévinas, nella risposta di Caino «vi è solamente ontologia: io sono io e lui è lui. Noi siamo esseri ontologicamente separati» ${ }^{17}$.

Ma la relazione fraterna porta con sé la responsabilità di riconoscere e accettare l'altro nella sua diversità. Non poggia, dunque, sul valore di ciò che si scambia; piuttosto esprime un rapporto di co-appartenenza il cui valore risulta intrinseco all'esistenza di ciascuno, a prescindere dal ruolo e dalla funzione sociale che si riveste.

Dopo aver ucciso Abele, Caino capisce di aver compiuto una violazione di carattere assoluto: «chiunque mi incontrerà mi potrà uccidere» (Gen 4, 14). «Ma Dio impone un segno a Caino, per impedire a chiunque di colpirlo» (Gen, 4, 15). Abele chiamato "il giusto", genera col proprio sacrificio il piacere del divino; il fratello l'antitesi. Si prefigura dunque il gradimento del sacrificio quale atto precursore alla generazione della figura cardine dell'agape e mediatore della trasposizione della vendetta con la riconciliazione.

Come raccontato dalla Genesi, Caino divenne costruttore di una città, ossia fondatore della vita politica, dello spazio pubblico. La stirpe

16 Cfr. BAGGIO, A. M. La fraternità antagonista. L'interpretazione freudiana e la fondazione della società egualitaria e conflittuale, In: Caino e $i$ suoi fratelli. II fondamento relazionale nella politica e nel diritto, a cura di A.M. Baggio, Città Nuova, Roma 2012, pp. 24-25.

17 LÉVINAS, E. Tra noi. Saggi sul pensare all'altro, Jaca Book, Milano 1998, p. 145. 
di Caino, dunque, risulta composta da uomini che si sono uniti politicamente proprio perché non devono uccidersi.

«La vita della città, cioè la vita politica, viene qui presentata come una seconda possibilità, offerta a colui che ha ucciso il fratello, di vivere la fraternità: la politica è il recupero e lo sviluppo del legame di fraternità, vivendolo non più attraverso un rapporto diretto e immediato (come fratelli di sangue), ma attraverso la mediazione della legge, cioè come cittadini.» ${ }^{18}$

Nonostante il modello ebraico sia assunto dal cristianesimo, la fraternità cainita viene ritenuta incapace di raggiungere gli scopi prospettati. E infatti Sant'Agostino farà della città cainita il tipo della "città terrena".

In epoca moderna abbiamo visto come sia forte il desiderio di riuscire a dar vita a società politiche che rifiutano il principio di autorità paterna; basti pensare alle due opere di Freud, Totem e tabù ${ }^{19}$, L'uomo Mosè e la religione monoteistica ${ }^{20}$. Ma rifiutando l'autorità paterna, anche la stessa dimensione pubblica della fraternità di cui abbiamo parlato finora verrebbe meno.

Nelle società contemporanee sembra essere sparita non solo la figura paterna, una volta immediatamente riconoscibile attraverso la figura del re, ma anche la dimensione paterna, «cioè la dimensione dell' autorità come distinta dal potere, una dimensione di cui ogni società continua ad avere bisogno, anche in assenza della figura del padre-re» ${ }^{21}$.

Per analizzare più a fondo la dimensione paterna occorre anzitutto operare una distinzione tra autorità e potere. Per farlo, basta immaginare che un genitore conserva la sua autorità anche quando non ha più potere sui figli.

18 BAGGIO, A. M. La fraternità antagonista. L'interpretazione freudiana e la fondazione della società egualitaria e conflittuale, In: Caino e i suoi fratelli. II fondamento relazionale nella politica e nel diritto, a cura di A. M. Baggio, Città Nuova, Roma 2012, p. 28.

19 FREUD, S. Totem e Taboo, (1913). Opere di Sigmund Freud (1912-1914). Opere, VII, Torino, Bollati Boringhieri, 2000.

20 FREUD, S. L'uomo Mosè e la religione monoteistica: tre saggi (1938). Opere di Sigmund Freud (1934-1938). Opere, XI, 1930/1938, Torino, Bollati Boringhieri, 2003, p. 331-461.

21 BAGGIO, A. M. La fraternità antagonista. L'interpretazione freudiana e la fondazione della società egualitaria e conflittuale, In: Caino e i suoi fratelli. II fondamento relazionale nella politica e nel diritto, a cura di A. M. Baggio, Città Nuova, Roma 2012, p. 53. 
Relazione fraterna e complesso fraterno arcaico possono essere considerati, nell'esperienza psicologica, espressione di un evitamento del complesso edipico ${ }^{22}$, con una precisa economia, dinamica, desiderio amoroso e narcisistico oggettuale rispetto alla rappresentazione percepita come fraterna.

Si tratta, dunque, della produzione di uno spazio psichico ospitante le rappresentazioni fantasmatiche della corporeità materna, che si declinano alle necessità di ammissione dell'esistenza dell'alterità fraterna. Ciò risulta sorretto dalla rivalità nell'investimento pulsionale, tipicamente edipica, che comporta uno stato di confusione rappresentazionale con la corporeità fantasmatica materna. Si comprende come, in questo senso, l'alterità possa essere vissuta, dal momento che il preedipico non prevede la piena considerazione della terzeita ${ }^{23}$ e di tutto il valore simbolico polisemico che ne scaturisce ${ }^{24}$.

Secondo le parole di Baggio, «avere autorità significa custodire un disegno, conservare i principi e i valori fondanti la vita della comunità o di un gruppo e, proprio per questo, mantenerne chiaro il fine e l'orientamento. Così come un genitore mantiene chiaro dentro di sé il valore e l'identità di un figlio, allo stesso modo l'autorità conserva l'identità, i valori fondanti, la ragion d'essere di un popolo. Per questo, solo il rapporto con l'autorità permette di conservare l'identità ${ }^{25}{ }_{\text {». }}$.

Il disprezzo per il padre e il conseguente rifiuto dell'autorità comporta che la società si consegni alle dinamiche di potere, non riuscendo più ad orientarlo verso gli scopi identitari che soltanto l'autorità è capace di individuare.

Arrivati a questo punto risulta più semplice comprendere come, una volta negato il padre, non è neanche più possibile considerarsi fratelli.

Non considerandosi fratelli «non si tollera più la differenza, ma si diviene antagonisticamente identici. La negazione del padre rende infatti impossibile ai fratelli di riconoscersi come tali e, dunque, di ammettere

22 KAËS, R. (2008). Le complexe fraternel archaïque, Revue française de psychanalyse, 72(2), 383-396.

23 PEIRCE, C.S. (1931-1958) Collected papers, Harvard University Press.

24 SETTINERI, S.; MERLO, E. M.; TURIACO, F., \& MENTO, C. (2017). The symbol theory in S. Freud, CG Jung and CS Peirce, Mediterranean Journal of Clinical Psychology, 5(2).

25 BAGGIO, A. M. La fraternità antagonista. L'interpretazione freudiana e la fondazione della società egualitaria e conflittuale, In: Caino e i suoi fratelli. II fondamento relazionale nella politica e nel diritto, a cura di A.M. Baggio, Città Nuova, Roma 2012, p. 54. 
e accettare il contenuto specifico della relazione di fraternità: la loro diversità nella parità filiale. La differenza è davvero vissuta in maniera malata $^{26} \gg$.

Non a caso, l'invidia rivela la presenza di una difficoltà identitaria, la difficoltà assimilativa con la paternità che genera il difetto identificativo del Sé e, di conseguenza, le difficoltà di articolazione nei rapporti paritari secondari; laddove la primità e la secondità collassano, la terzeità risulta negata. La fenomenologia dell'invidia diviene trasversale modalità di articolazione delle relazioni con l'Altro, che impregna il soggetto, dominandone lo spazio interno emotivo, l'affettività derivante e le immagini orbitanti attorno. Dunque si genera la supremazia di quei fenomeni intrapsichici, i complessi, la cui potenza e i cui esiti disadattivi dipendono direttamente dallo spazio arrogato e dall'intrusività che occorre nella relazione d'oggetto.

Dal punto di vista psicologico, un'importante approvazione di quanto detto viene dagli studi della Klein ${ }^{27}$, che illustra lo sviluppo dell'invidia da un punto di vista complementare alla paternità, cioè la maternità.

«Il soggetto sano, realmente adulto e maturo, capace di superare gli ostacoli costruiti dall'invidia, dalla gelosia, dall'avidità, conserva sempre la "forma" del figlio, ed è questo ciò che gli permette di essere fratello, cioè di costruire positivi legami orizzontali con i propri pari ${ }^{28}{ }_{\text {》. }}$.

La pressione identificatoria è considerevole. Si ritrovano le figure di fratelli e sorelle in tutti gli investimenti pulsionali. Poiché non esiste differenza generazionale, fratelli e sorelle caratterizzano, più dell'identificazione coi genitori, le scelte esistenziali a livello dell'Io. I rapporti tra fratelli e sorelle costituirebbero una « funzione », svolgendo un ruolo di legame di passaggio, mediatore tra famiglia e società ${ }^{29}$.

26 BAGGIO, A. M. La fraternità antagonista. L'interpretazione freudiana e la fondazione della società egualitaria e conflittuale, In: Caino e i suoi fratelli. II fondamento relazionale nella politica e nel diritto, a cura di A. M. Baggio, Città Nuova, Roma 2012, p. 58.

27 KLEIN, M., TOLENTINO, L. Z., \& SARAVAL, A. (1969). Invidia e gratitudine. Firenze: Martinelli.

28 BAGGIO, A. M. La fraternità antagonista. L'interpretazione freudiana e la fondazione della società egualitaria e conflittuale, in Caino e $i$ suoi fratelli. II fondamento relazionale nella politica e nel diritto, a cura di A. M. Baggio, Città Nuova, Roma 2012, p. 61.

29 CAMUS-DONNET, D. (2008). La fonction fraternelle. Revue française de Psychanalyse, 72(2), 409-417. 
Risulta piuttosto plausibile che, all'inizio, questa funzione potrebbe essere concepita come un'estensione delle questioni edipiche e il loro sorpasso nella direzione di strutturare il Super-Io post-Edipico e gli ideali relativi al periodo di latenza.

\section{La fraternità come possibilità del divenire}

Attraverso il paradigma della fraternità universale viene spiegata la dinamica trinitaria cristiana, esempio di terzeità e di integrazione dell'alterità dal punto di vista simbolico e psicologico. A partire dal sacrificio cristiano, non c'è più bisogno di ricorrere necessariamente alla violenza del capro espiatorio. L'autorità consegnataci dal paradigma trinitario lascia l'uomo libero di sperimentarsi nelle relazioni sociali. Tale paradigma mette a nudo il fatto che l'identità di Uno si rivela nella relazione con l'Altro.

L'esempio dell'origine offertoci dal mito, e l'occasione di fornire significati offertaci dalla visione teleologica religiosa, confrontati con la clinica e la teoria psicologica dinamica, ci consentono di estendere il senso, tale è la trasversalità del tema. La disseminazione del rapporto fraterno, tra il registro edipico e quello pre-edipico, mostra come esso sia un perno per l'analisi, tanto dei legami primari quanto di quelli edipici ${ }^{30}$.

Il ruolo protettivo del legame fraterno e la sua riattivazione transferenziale consente una progressiva decondensazione di immagini genitoriali negative, permettendo di affrontare un rapporto materno primario carente. La diffrazione dei fantasmi originari attraverso le figure del legame fraterno permette di dar loro una forma protettiva. Da questo punto di vista è possibile intendere la fraternità come una possibilità progressiva di mitigazione del conflitto, sia esso intrapsichico che interpsichico.

L'isolamento dell'uomo nella propria mente, tutto ciò che egli pensa, sente, o percepisce, è una rappresentazione. Da questo punto di vista, l'esistenza del mondo è possibile nella misura in cui la mente produce un'immagine di esso $^{31}$.

30 PARAT, H. (2008). La relation fraternelle entre vœux œdipiens et plaintes pré-œdipiennes, Revue française de Psychanalyse, 72(2), 419-434.

31 JUNG, C. G. (1954). The Tibetan Book of the Great Liberation, Or, The Method of Realizing Nirvānna Through Knowing the Mind: Preceded by an Epitome of Padma-Sambhava's Biography, Oxford University Press, USA. 
Le immagini, considerabili per loro natura come una forma di $\operatorname{coscienza}^{32}$, giacché i fenomeni fisici sono vissuti dal soggetto e percepiti attraverso $i$ cinque sensi, diventano fenomeni psichici e rappresentazioni appartenenti alla coscienza del soggetto ${ }^{33}$.

In tal senso, la limitatezza dell'esperienza propria della mente suggerisce essa stessa la necessità di mediazione attraverso oggetti psichici in grado di produrre un adattamento progressivo con l'alterità e la rilevanza che la qualità dei fenomeni d'immagine possano assumere, specialmente laddove l'investimento affettivo risulta massivo. Come è chiarito dalla pratica psicodinamica, la successione e il passaggio dalla primità alla secondità, e l'integrazione della terzeità, costituiscono delle potenti fenomenologie intrapsichiche attraverso cui la psiche darà rapporto col mondo. La visione analitica promuove un aspetto di fondamentale rilevanza per la considerazione sana dell'alterità.

Ed è per questo che la limitatezza dell'esperienza propria della mente suggerisce la necessità di mediazione attraverso oggetti psichici in grado di produrre un adattamento progressivo con l'alterità e la rilevanza che la qualità dei fenomeni d'immagine possono assumere, specialmente laddove l'investimento affettivo risulta massivo.

\section{Conclusioni}

Come è chiarito dalla pratica psicodinamica, la successione e il passaggio dalla primità alla secondità e l'integrazione della terzeità, costituiscono delle potenti fenomenologie intrapsichiche attraverso cui la psiche darà rapporto col mondo.

La visione analitica promuove un aspetto di fondamentale rilevanza per la considerazione sana dell'alterità.

Suggerisce Jung nella simbolica dello spirito: «per designare l'orizzonte, quattro punti cardinali; in natura, quattro elementi; quattro colori; quattro qualità primitive; quattro caste in India; quattro vie di sviluppo spirituale nel buddhismo; quattro aspetti significativi dell'orientamento psichico, ecc. La completezza è il cerchio, il manda-

32 SARTRE, J. P. L'imaginaire: psychologie phénomenologique de l'imagination (1940), trad. it. L'immaginario. Psicologia fenomenologica dell'immaginazione. (2007) Piccola Biblioteca Einaudi, Torino.

33 HUSSERL, E. (1911). "Philosophie als strenge Wissenschaft", in: Logos; trad. it.: La filosofia come scienza rigorosa, Laterza, Bari, 2005, p. 3-4. 
la, e la sua minima divisione naturale è la quaternità ${ }^{34} \gg$. Tale considerazione prevede la presa di coscienza di una trasversalità che è tipica dell' inconscio collettivo, laddove l'elemento fortemente umano e naturale coincidono nell'immagine condivisa, la cui economia governa l'energia della psiche, sia essa intesa in termini creativi, sia essa invece garantita dall'egemonia del complesso.

La totalità raggiungibile attraverso la considerazione dell'ultimo termine quaternario, rispetto ai precedenti terzeitari, costituisce la possibilità di completamento che è alla base del cammino del processo individuativo. In tal senso, la possibilità di integrazione dell'ombra ${ }^{35}$ costituirebbe non la conclusione, ma la conditio sine qua non dell'avvio del processo. La fraternità, in tal senso, costituirebbe, attraverso l'occasione di considerazione e non di negazione della propria ombra insita solo proiettivamente nell'Altro, l'occasione di incontro con l'immagine, il cui moto reattivo della semplice proiezione confermerebbe la natura dell'ombra senza mai occasionarne il ritorno. Prendersi del tempo per riflettere su tali argomentazioni, in qualche modo, può favorirci a scavare all'interno della nostra interiorità e a farci riflettere sul valore imprescindibile che custodisce l'ammissione incondizionata dell'altro in quanto tale.

\section{Bibliografia}

BAGGIO, A. M. La fraternità antagonista. L'interpretazione freudiana e la fondazione della società egualitaria e conflittuale, In: Caino e $i$ suoi fratelli. Il fondamento relazionale nella politica e nel diritto, a cura di A.M. Baggio, Città Nuova, Roma 2012, p. 19-61.

BRUSSET, A. La lien fraternel et la psychanalyse, Psychanalyse à l'Universitè, 12, n. 45, Paris, AUREPP, 1987.

BRUSSET, B. (2008). Le lien fraternel et la psychanalyse. Revue française de psychanalyse, 72(2), 347-382.

CAMUS-DONNET, D. (2008). La fonction fraternelle. Revue française de Psychanalyse, 72(2), 409-417.

JUNG, C.G. La simbolica dello spirito, Torino, Boringhieri 1959.

34 JUNG, C. G. La simbolica dello spirito, Torino, Boringhieri 1959, p. 245.

35 JUNG, C. G. Saggio d'interpretazione psicologica del dogma della Trinità, in Jung, Opere, Vol. 11, Psicologia e religione, Torino, Boringhieri 1979. 
JUNG, C.G. Saggio d'interpretazione psicologica del dogma della Trinità, in Jung, Opere, Vol. 11, Psicologia e religione, Torino, Boringhieri, 1979.

LÉVINAS, E. Tra noi. Saggi sul pensare all'altro, Jaca Book, Milano 1998.

FREUD, S. Totem e Taboo, (1913). Opere di Sigmund Freud (1912-1914). Opere, VII, Torino, Bollati Boringhieri, 2000.

FREUD, S. L'uomo Mosè e la religione monoteistica: tre saggi (1938). Opere di Sigmund Freud (1934-1938). Opere, XI, 1930/1938, Torino, Bollati Boringhieri, 2003, p. 331-461.

HUSSERL, E. (1911). "Philosophie als strenge Wissenschaft”, in: Logos; trad. it.: La filosofia come scienza rigorosa, Laterza, Bari, 2005, 3-4.

JUNG, C. G. Considerazioni generali sulla Teoria dei Complessi (1934). Opere di Jung, vol. 8.1976, Torino, Bollati, Borringhieri.

JUNG, C. G. (1954). The Tibetan Book of the Great Liberation, Or, The Method of Realizing Nirvanna Through Knowing the Mind: Preceded by an Epitome of Padma-Sambhava's Biography, Oxford University Press, USA.

JUNG, C. G. K. (1948). Karölyi, Prolegomeni allo studio scientifico della mitologia, trad. it. Torino, Einaudi.

KAËS, R. (2008). Le complexe fraternel archaïque, Revue française de psychanalyse, 72(2), 383-396.

KLEIN, M.; TOLENTINO, L. Z.; \& SARAVAL, A. (1969). Invidia e gratitudine. Firenze: Martinelli.

LACAN, J. (1932), De la psychose paranoiaque dans ses rapports avec la personalitè, Paris, Le Francoise ; rééd. Paris, Le Seuil, 1975.

LECHARTIER-ATLAN, C. (2008). Frères et sœurs: une introduction. Revue française de psychanalyse, 72(2), 331-338.

MALLET, J. (2008). Une théorie de la paranoïa. Revue française de psychanalyse, 72(2), 341-346.

PARAT, H. (2008). La relation fraternelle entre voux oedipiens et plaintes pré-oedipiennes. Revue française de Psychanalyse, 72(2), 419-434.

PEIRCE, C.S. (1931-1958), Collected papers, Harvard University Press. 
RECALCATI, M. (2012). Ritratti del desiderio, Raffaello Cortina Editore.

SARTRE, J. P. L'imaginaire: psychologie phénomenologique de l'imagination (1940), trad. it. L'immaginario. Psicologia fenomenologica dell'immaginazione. (2007) Piccola Biblioteca Einaudi, Torino.

SETTINERI, S.; MERLO, E. M.; TURIACO, F.; \& MENTO, C. (2017). The symbol theory in S. Freud, CG Jung and CS Peirce. Mediterranean Journal of Clinical Psychology, 5(2). 